\title{
Genome-wide expression profiling and functional characterization of SCA28 lymphoblastoid cell lines reveal impairment in cell growth and activation of apoptotic pathways
}

Cecilia Mancini ${ }^{1}$, Paola Roncaglia ${ }^{2,3}$, Alessandro Brussino ${ }^{1}$, Giovanni Stevanin ${ }^{4,5,6}$, Nicola Lo Buono ${ }^{1}$, Helena Krmac ${ }^{3}$, Francesca Maltecca ${ }^{7}$, Elena Gazzano ${ }^{8}$, Anna Bartoletti Stella ${ }^{9}$, Maria Antonietta Calvaruso ${ }^{10,11}$, Luisa Iommarini ${ }^{10}$, Claudia Cagnoli ${ }^{1}$, Sylvie Forlani ${ }^{4}$, Isabelle Le Ber ${ }^{4}$, Alexandra Durr ${ }^{4,5}$, Alexis Brice ${ }^{4,5}$, Dario Ghigo ${ }^{8}$, Giorgio Casari ${ }^{7}$, Anna Maria Porcelli ${ }^{10}$, Ada Funaro ${ }^{1}$, Giuseppe Gasparre ${ }^{9}$, Stefano Gustincich ${ }^{3}$ and Alfredo Brusco ${ }^{1,11^{*}}$

\begin{abstract}
Background: SCA28 is an autosomal dominant ataxia associated with AFG3L2 gene mutations. We performed a whole genome expression profiling using lymphoblastoid cell lines (LCLs) from four SCA28 patients and six unrelated healthy controls matched for sex and age.
\end{abstract}

Methods: Gene expression was evaluated with the Affymetrix GeneChip Human Genome U133A 2.0 Arrays and data were validated by real-time PCR.

Results: We found 66 genes whose expression was statistically different in SCA28 LCLs, 35 of which were up-regulated and 31 down-regulated. The differentially expressed genes were clustered in five functional categories: (1) regulation of cell proliferation; (2) regulation of programmed cell death; (3) response to oxidative stress; (4) cell adhesion, and (5) chemical homeostasis. To validate these data, we performed functional experiments that proved an impaired SCA28 LCLs growth compared to controls $(p<0.005)$, an increased number of cells in the G0/G1 phase $(p<0.001)$, and an increased mortality because of apoptosis $(p<0.05)$. We also showed that respiratory chain activity and reactive oxygen species levels was not altered, although lipid peroxidation in SCA28 LCLS was increased in basal conditions $(p<0.05)$. We did not detect mitochondrial DNA large deletions. An increase of TFAM, a crucial protein for mtDNA maintenance, and of DRP1, a key regulator of mitochondrial dynamic mechanism, suggested an alteration of fission/fusion pathways.

Conclusions: Whole genome expression profiling, performed on SCA28 LCLs, allowed us to identify five altered functional categories that characterize the SCA28 LCLs phenotype, the first reported in human cells to our knowledge.

Keywords: Autosomal dominant cerebellar ataxia, Spinocerebellar ataxia, SCA28, AFG3L2, Genome-wide expression, LCLS

\footnotetext{
*Correspondence: alfredo.brusco@unito.it

'Department of Medical Sciences, University of Torino, via Santena 19,

10126 Torino, Italy

${ }^{11}$ Medical Genetics Unit, "Città della Salute e della Scienza" Hospital, Torino,

Italy

Full list of author information is available at the end of the article
} 


\section{Background}

Spinocerebellar ataxias or SCAs are a heterogeneous group of autosomal dominant neurodegenerative diseases with an incidence of $\sim 1: 30,000$ and an onset which is typically in adulthood. At least 30 different subtypes and 19 causative genes have been reported [1]. For all such SCA genes, despite a well-described clinical and pathological phenotype, the molecular and cellular events that underlie neurodegeneration are still poorly understood.

SCA28 is one of the more recently identified forms, and is associated with mutations in AFG3L2 (ATPase family gene 3-like 2) on chromosome 18p [2,3]. All mutations so far reported are missense changes, all are located in the M41-protease domain of the AFG3L2 protein with the exception of one (p.Asn432Thr) [3-5]. The disease prevalence is around $1.5 \%$ among SCA patients of European descent [2-4]. The AFG3L2 gene encodes a subunit of the ATP-dependent metalloprotease m-AAA (ATPases Associated with a variety of cellular Activities), located within the inner mitochondrial membrane [6], where it exerts different functions in mitochondrial proteins processing, maturation, activation and quality control, or degradation [7-12]. The m-AAA displays a hexameric ring structure that in humans can be a homo-oligomer formed by AFG3L2 only, or a hetero-oligomer formed by AFG3L2 and Paraplegin (a paralog of AFG3L2 encoded by the SPG7 gene, whose loss-of-function causes an autosomal recessive form of hereditary spastic paraplegia [12,13]). A homozygous AFG3L2 mutation (p.Tyr616Cys) has been detected in two children affected by an early-onset severe spastic ataxia-neuropathy syndrome, characterized by severe spasticity, ataxia, and myoclonic epilepsy [14]. A recent report of SPG7 dominant mutations in a form of optic atrophy shows that both genes may give dominant and recessive phenotypes [15].

Several groups have attempted to characterize the biochemical defects caused by alterations in the AFG3L2 protein, albeit no study on human cells has been reported thus far. Yeast cells lacking the m-AAA complex transfected with mutated AFG3L2 show a respiratory defect (i.e. incapacity to grow on a non-fermentable carbon source) related to a deficiency of respiratory chain complex IV and proteolytic impairment [3].

ATP production has been evaluated in the brain of both Afg3l2 par/par and Afg3l2 $2^{\text {Emv66/Emv6r }}$ mutant mice, which are a spontaneous mouse mutant (paralysé) carrying an Arg389Gly substitution in the conserved AAAdomain of AFG3L2 (Afg3l2 ${ }^{\text {par/par }}$ ), and a null Afg3l2 mouse model with a murine leukemia proviral insertion in exon $14\left(A f g 3 l 2^{E m v 66 / E m v 66}\right)$, respectively. In the presence of different substrates and inhibitors of the respiratory chain, results point to an assembly impairment of complexes I and III [16], while alterations in their activity and in ATP production were demonstrated in a heterozygous mouse $A f g 3 l 2^{+/ E m v 66}$, although with an onset at 4-6 months of age [17].

In this study, we characterized the genome-wide expression profile, and the cellular phenotype of human lymphoblastoid cell lines (LCLs) derived from SCA28 patients, highlighting alterations in specific pathways and functions correlated to the disease.

\section{Methods}

\section{Cells lines}

We obtained lymphoblastoid cell lines (LCLs) derived from SCA28 patients carrying the p.Gly671Arg $(n=3)$, p.Met666Arg $(\mathrm{n}=1)$, p.Met666Val $(\mathrm{n}=3)$, p.Met666Thr $(\mathrm{n}=2)$, and p.Thr654Ile $(\mathrm{n}=1)$ mutations. Clinical features of these patients have been previously described [4] and are summarized in [Additional file 1]. Fourteen control LCLs were obtained from the Human Genetics Foundation of Torino ( $\mathrm{HuGeF}$ ).

LCLs were grown at $37^{\circ} \mathrm{C}$ and $5 \% \mathrm{CO}_{2}$ in RPMI-1640 medium supplemented with $10 \%$ heat-inactivated fetal bovine serum, $2 \mathrm{mM}$ L-glutamine, $0.1 \mathrm{mg} / \mathrm{ml}$ streptomycin, and $100 \mathrm{U} / \mathrm{ml}$ penicillin.

\section{Whole genome RNA expression profiling}

Total RNA was extracted from $5 \times 10^{6}$ LCLs using the Qiaquick RNA Easy plus extraction kit (Qiagen, Mannheim, Germany) according to manufacturer's instructions. A sample of the total RNA was visualized in a $0.6 \%$ TBE 1X/agarose gel and its quality assessed using the Agilent BioAnalyser 2100 (Agilent, Palo Alto, CA). RNA was then quantified with a NanoDrop 1000 spectrophotometer (Thermo Scientific, Barrington IL, USA).

Whole-genome RNA expression study was performed on a subgroup of four SCA28 patients, each carrying a different AFG3L2 mutation (p.Thr654Ile, p.Met666Val, p. Met666Thr, and p.Gly671Arg). Six unrelated healthy subjects matched for sex and age at sampling were used as controls.

For each sample, total RNA (6 $\mu \mathrm{g}$ ) was labeled according to the standard one-cycle amplification and labeling protocol developed by Affymetrix (Santa Clara, CA, USA). Labeled cRNA was hybridized on Affymetrix GeneChip Human Genome U133A 2.0 Arrays containing probes for over 18,400 transcripts. Hybridized arrays were stained and washed on a GeneChip Fluidics Station 450 and then scanned on a GeneChip Scanner 3000 7G (Affymetrix). Cell intensity values and probe detection calls were computed from the raw array data using the Affymetrix GeneChip Operating Software (GCOS). Further data processing was performed in the $\mathrm{R}$ computing environment using specific packages from the BioConductor software project [18]. Robust Multi-Array Average (RMA) normalization was applied. Normalized data were filtered based on the Affymetrix detection call, so that only probes 
that had a Present call in at least one of the arrays were retained. Probes with low intensity values (less than 100) in all arrays were also excluded from statistical analysis.

Data were imported into the MultiExperiment Viewer $(\mathrm{MeV})$ software [19], and statistical analysis was performed to detect significantly differentially expressed genes in SCA28 patients versus healthy controls, using two different tests as implemented within the MeV: Rank Product (RP) [20] and Significance Analysis of Microarrays (SAM) [21]. RP was run using 100 permutations and a False Discovery Rate (FDR) of $0.005 \%$. The SAM test was run using 1000 permutations and a FDR of 3\%. Differentially expressed genes were then specifically examined based on their Gene Ontology annotations [22] and through the use of the Database for Annotation, Visualization and Integrated Discovery (DAVID) Bioinformatics Resources [23-25].

\section{Validation of data by real-time RT-PCR}

Genes to be validated were selected on the basis of three criteria: (i) an expression level higher than 7, (ii) fold changes higher than 1.5 or lower than 0.7 in patients $v s$. controls, and (iii) the potential interest of the gene in SCA28 pathogenesis as inferred by its function, the pathway in which it was involved and/or the functional similarity with known ataxia-related genes.

Quantitative real time PCR was used to validate array findings for 11 genes in the same LCLs batches used for microarray expression profiling (see above). The validated genes were then tested using a different RNA extraction from the same subjects (4 patients vs. 6 controls) to obtain a technical replicate. In addition, a third real-time PCR experiment on the same genes was performed on LCLs generated from four new different SCA28 patients obtained later [mutations p.Met666Arg, p.Met666Thr and p.Met666Val $(n=2)]$, as biological replicate.

Total RNA was extracted from eight SCA28 patients and eight healthy controls as described above, and $1 \mu \mathrm{g}$ was retrotranscribed using the Transcription First Strand cDNA synthesis kit (Roche Diagnostics, Mannheim, Germany). Quantitative real-time RT-PCRs were carried out on an ABI-Prism7500 Fast instrument (Applied Biosystems) using the Taqman Gene Expression Master Mix (Applied Biosystems), Universal Probe Library (UPL) technology (Roche) and according to the manufacturer's protocol. Primers and UPL probes used for real time PCR validation are listed in Additional file 2.

Experimental $\mathrm{Ct}$ values were normalized to TATAbinding protein, used as endogenous control (VIC labeled pre-designed TaqMan gene expression assays, TBP, Hs00427620_m1, Applied Biosystems). Gene expression was calculated in each sample relative to the mean of controls, using the delta-delta $\mathrm{Ct}$ method as described elsewhere [26]. Each sample was examined in triplicate and differences in gene expression of patients relative to controls were statistically evaluated by the Student's $t$-test for at least three independent experiments.

\section{Cell growth analysis}

Cell growth was determined using the MTT test [Carbonyl cyanide $\mathrm{p}$-(trifluoromethoxy) phenylhydrazone, 3-(4,5-dimethylthiazol) 2,5- diphenyl-tetrazolium bromide, Sigma-Aldrich] [27]. The growth of LCLs from six patients carrying the p.Thr654Ile $(\mathrm{n}=1)$, p.Met666Thr $(\mathrm{n}=1)$, p.Met666Val $(\mathrm{n}=2)$, p.Met666Arg $(\mathrm{n}=1)$ and p.Gly671Arg $(n=1)$ mutations was compared to the growth of seven LCLs from healthy controls, performing six replicates for each of the five time-points analyzed $(0,3$, 6, 24, and 48 hours). After overnight incubation in RPMI with $0.5 \%$ FCS, $3 \times 10^{4}$ cells for each time-point were washed twice in PBS solution, the pellet was resuspended in $1 \mathrm{ml}$ of RPMI supplemented with 10\% FCS and seeded onto 96-well plates $(150 \mu \mathrm{l} /$ well $)$. At each time-point, $10 \mu \mathrm{l}$ of a $5 \mathrm{mg} / \mathrm{ml} \mathrm{MTT} \mathrm{solution} \mathrm{were} \mathrm{added} \mathrm{to} \mathrm{each} \mathrm{well.} \mathrm{A}$ negative control was included in each experiment to exclude microbial contamination.

After 1 hour incubation at $37^{\circ} \mathrm{C}$ in the dark the MTT solution was carefully removed, and cells were dissolved in $100 \mu \mathrm{L}$ of DMSO for 10 minutes at $37^{\circ} \mathrm{C}$. Proliferation rate of cells was determined by measuring the optical density (OD) of each sample at $570 \mathrm{~nm}$ with an ELISA Microplate Reader Model 680 (Biorad). To confirm growth curves with a second method, independent of mitochondrial function, we used a cytoplasmic membrane-dye (Neuro-DiO, Biotium) whose fluorescence intensity decreases as a consequence of cell divisions. The design of the experiment was the same as that of the MTT assays, and fluorescence was analyzed by a FACS Calibur flow cytometer using CellQuest software (Becton Dickinson, USA).

\section{Analysis of cell cycle}

Cell cycle was analyzed by fluorescence labeling of DNA with Propidium Iodide (PI) [28], a stoichiometric dye, that binds in proportion to the amount of DNA present in the cell. LCLs from six healthy controls and six SCA28 patients carrying the p.Thr654Ile $(\mathrm{n}=1)$, p.Met666Thr $(\mathrm{n}=1)$, p.Met666Val $(\mathrm{n}=2)$, p.Met666aArg $(\mathrm{n}=1)$, and p.Gly671Arg $(n=1)$ mutations were compared at three different time-points $(0,3$, and $24 \mathrm{~h})$. Approximately $5 \times$ $10^{5}$ cells for each time-point were subjected to density gradient centrifugation Ficoll-Paque Plus (GE Healthcare, Piscataway, NJ) to remove any dead cell and debris. Viable cells were collected at the interface and washed with PBS solution. Cells were synchronized by overnight incubation in RPMI containing 0.5\% FCS, washed twice in PBS and seeded onto a 24-wells plate in $0.5 \mathrm{ml}$ of RPMI $10 \%$ FCS at the final concentration of $5 \times 10^{5} /$ well. At the designed time-points (0,3 and $24 \mathrm{~h}$ ), each well was washed with PBS and resuspended in $1 \mathrm{ml}$ of PBS with 
$0.1 \%$ BSA. One $\mathrm{ml}$ of $70 \%$ Ethanol was added to each sample for 45 minutes at $4^{\circ} \mathrm{C}$, samples were then rinsed with PBS, suspended in $0.5 \mathrm{ml}$ of PBS with $0.1 \%$ BSA and $5 \mu \mathrm{l}$ of RNaseA [10 mg/ml], and incubated 30 minutes at $37^{\circ} \mathrm{C}$. One $\mu \mathrm{l}$ of PI was added to each sample for $10 \mathrm{mi}-$ nutes at $4^{\circ} \mathrm{C}$ in the dark, then cells populations were analyzed on a FACS Calibur flow cytometer using CellQuest software (Becton Dickinson).

\section{Analysis of apoptosis}

Apoptosis was evaluated by fluorescence-activated cell sorting (FACS) after Annexin V/propidium iodide (PI) double staining (Biovision, San Francisco, CA, USA). LCLs from six healthy controls and six patients carrying the p.Thr654Ile $(\mathrm{n}=1)$, p.Met666Arg $(\mathrm{n}=1)$, p.Met666Thr $(\mathrm{n}=1)$, p.Met666Val $(\mathrm{n}=2)$, and p.Gly671Arg $(\mathrm{n}=1)$ mutations were used [29].

Cells were washed twice with Annexin-Binding Buffer $(\mathrm{ABB})$ [30], and incubated for 5 minutes in the dark with: 1) ABB alone; 2) Annexin V-FITC; 3) PI; 4) Annexin V-FITC and PI. Early apoptotic cells were only stained by Annexin V-FITC, whereas late apoptotic or necrotic cells were double-stained by Annexin V-FITC and PI. Cells were analyzed on a FACS Calibur flow cytometer using CellQuest software (Becton Dickinson). Cells in which apoptosis was induced by overnight incubation with $1 \mu \mathrm{M}$ staurosporine were used as the positive control. To exclude that increasing apoptosis was due to starvation, the same experiments were performed without overnight incubation in RPMI with $0.5 \%$ FCS.

\section{ATP synthesis and respiratory chain complex evaluation}

Mitochondria were isolated from three SCA28 and three controls LCLs by differential centrifugation as described [31]. Ten million cells were homogenized using a glassTeflon homogenizer in an isotonic buffer $[0.25 \mathrm{M}$ sucrose, $20 \mathrm{mM}$ 3-(N-morpholino) propanesulfonic acid (MOPS), pH 7.2, $1 \mathrm{mM}$ EDTA] supplemented with $0.1 \%$ BSA and $0.1 \mathrm{mg} / \mathrm{ml}$ digitonin. Cell debris and nuclei were pelleted twice by centrifugation at 2,500 $\mathrm{g}$ for $5 \mathrm{mi}$ nute at $4^{\circ} \mathrm{C}$. Supernatants were centrifuged at $12,000 \mathrm{~g}$ for $25 \mathrm{~min}$ at $4^{\circ} \mathrm{C}$ and the mitochondrial pellet was resuspended in isotonic buffer $(0.5 \mathrm{M}$ sucrose, $20 \mathrm{mM}$ MOPS, pH 7.2, 1 mM EDTA).

Respiratory chain activity was evaluated using a protocol previously described [16] and adapted to LCLs. In brief, isolated mitochondria were incubated at $37^{\circ} \mathrm{C}$ for $30 \mathrm{~min}$ in a respiratory buffer $(0.25 \mathrm{M}$ sucrose, $20 \mathrm{mM}$ MOPS, $1 \mathrm{mM}$ EDTA, $5 \mathrm{mM}$ inorganic phosphate, $0.1 \%$ BSA, and $1 \mathrm{mM}$ ADP, $\mathrm{pH}$ 7.4). The function of each respiratory chain complex was tested by providing buffers containing specific substrates and inhibitors.

(1) Buffer 1 (5 $\mathrm{mM}$ pyruvate and $1 \mathrm{mM}$ malate) and (2) buffer 2 (5 mM glutamate and $1 \mathrm{mM}$ malate) were used to stimulate complex I, II, III, IV, and V-dependent ATP synthesis. (3) Buffer 3 containing a complex I inhibitor (1 $\mathrm{mM}$ rotenone) and $10 \mathrm{mM}$ succinate, was used to measure ATP production that is dependent on complex II, III, IV, and V. Finally, (4) buffer 4 [2 mM antimycin A -inhibitor of complex III- and $0.1 \mathrm{mM}$ tetramethylp-phenylenediamine (TMPD) / $2 \mathrm{mM}$ ascorbate] was used to evaluate the ATP level resulting from the activity of complex IV and V.

ATP concentration was determined with the luciferinluciferase method as described previously [32]. In brief, isolated mitochondria were lysed with the ATP lysis buffer (200 mM NaOH and $500 \mathrm{nM}$ EDTA), and an aliquot of the obtained extract was diluted with the ATP dilution buffer (100 mM NaOH and $500 \mathrm{nM}$ EDTA). Twenty microliters of this mixture were added to $100 \mu$ of the assay solution (250 mM glycylglycine, $2 \mathrm{mM}$ EGTA, $2 \mathrm{mM}$ $\mathrm{MgCl}_{2}, 0.4 \mathrm{~g} / \mathrm{L} \mathrm{BSA}, 7.5 \mathrm{mM}$ DTT, $15 \mu \mathrm{M}$ luciferin, and 10 $\mu \mathrm{g} / \mathrm{ml}$ luciferase), and the ATP content was measured using a luminometer. Three independent experiments were performed, and the significance of the means calculated using two-tailed Student's $t$-test.

\section{Quantification of total cellular ATP levels}

The amount of cellular ATP was measured by using the Adenosine 5'-triphosphate (ATP) Bioluminescent Assay Kit (Sigma-Aldrich, St. Louis, MO, USA), as described previously [33]. ATP content was measured in duplicate, according to manufacturer's instructions, using an appropriate internal ATP standard for calibration. Total protein quantification was performed using classical Bradford protocol.

\section{Thiobarbituric acid reactive substances (TBARS) measurement}

Lipid peroxidation was evaluated measuring the Thiobarbituric acid reactive substances (TBARS) levels, following published methods [34]. Cells were washed twice in PBS and the pellet resuspended in $600 \mu \mathrm{l}$ of PBS. Five $\mu \mathrm{l}$ of Triton X-100 and $500 \mu \mathrm{l}$ of "TBA solution" (0.375\% Thiobarbituric acid and 30\% Trichloroacetic Acid in $0.5 \mathrm{~N} \mathrm{HCl}$ ) were added to $500 \mu \mathrm{l}$ of cells. Samples were boiled at $100^{\circ} \mathrm{C}$ for 20 minutes and quickly cooled by immersion in an ice bath, and centrifuged at $13000 \mathrm{~g}$ for 5 minutes. The absorbance of $300 \mu \mathrm{L}$ of supernatants was read at $532 \mathrm{~nm}$ with a Synergy HT microplate reader (Bio-Tek Instruments, Winooski, VT), reading the absorbance at $532 \mathrm{~nm}$. TBARS values were expressed as $\mathrm{nmol} / \mathrm{mg}$ proteins.

\section{Reactive Oxygen Species evaluation}

Intracellular Reactive Oxygen Species (ROS) levels were determined by using the fluorescent dye, DCFH-DA, 2,7-dichlorodihydrofluorescein diacetate $(5 \mu \mathrm{M})$. DCFH 
reacts with ROS to generate a new highly fluorescent compound, dichlorofluorescein, which can be analyzed by flow cytometry. Cells were incubated with DCFH-DA $(5 \mu \mathrm{M})$ at $37^{\circ} \mathrm{C}$ for $30 \mathrm{~min}$, washed twice with PBS, and then measured on a FACS Calibur flow cytometer using CellQuest software (Becton Dickinson). Cells treated with $\mathrm{H}_{2} \mathrm{O}_{2}(10 \mu \mathrm{M})$ were used as the positive control. Three independent experiments were performed.

\section{Mitochondrial DNA analysis}

Evaluation of mtDNA copy number was performed with an absolute quantitative TaqMan real-time PCR using the LightCycler480 (Roche), as previously detailed [35]. Long-range PCR was performed to screen for the presence of mtDNA deletions or rearrangements, as described [36].

\section{Western blot}

Total proteins were extracted from LCLs of four patients and four healthy controls, using the RIPA Lysis Buffer [TrisHCl $(50 \mathrm{mM}), \mathrm{NaCl}(150 \mathrm{mM})$, Triton (1\%)] and quantified using standard Bradford protocol. Proteins $(20-40 \mu \mathrm{g})$ were denaturated for $5 \mathrm{~min}$ at $99^{\circ} \mathrm{C}$ in Laemmli Buffer (Biorad) and separated by SDS-PAGE (10\% or 12\%) and transferred to nitrocellulose membranes (Biorad) in Tris-Glycine Buffer with 20\% methanol. Western Breeze Kit (Invitrogen) was used for blocking, hybridization, secondary antibodies, and detection of antibodies, following manufacturer's instructions. Hybridizations were performed with primary antibodies against POLY (Abcam, ab116049, 1:1000), TFAM (Abcam, ab131607, 1:1000), VDAC (Abcam, ab15895, 1:700), DRP1 (BD Biosciences, \#611112, 1:1000), OPA1 (BD Biosciences, \#612606, 1:1000), MFN1 (Abcam, ab57602, 1:1000), MFN2 (Abcam, ab56889, 1:1000), ATPase- $\alpha$ (BD Bioscience, \#612516, 1:1000) and NDUFA9 (LifeSpan Bioscience, LS-C133337 1:1000). Beta-actin antibody (Abcam Ab8227, 1:2000) or Lamin B1 antibody (Abcam, ab16048, 1:6000) were used as loading controls.

Part of the Western blot assays (DRP1, OPA1, MFN1, and MFN2) were performed after synchronization of the cells, obtained through overnight incubation in RPMI with $0.5 \%$ FCS followed by serum replacement with RPMI medium supplemented with $10 \%$ FCS for 24 hours.

\section{Results}

\section{Whole genome expression analysis in SCA28}

\section{lymphoblastoid cells}

To identify pathways associated with SCA28, we analyzed whole genome expression profiles in four patients' LCLs carrying different missense mutations [p.Thr654Ile $(\mathrm{n}=1) ;$ p.Met666Thr $(\mathrm{n}=1) ;$ p.Met666Val $(\mathrm{n}=1)$; p.Gly671Arg $(\mathrm{n}=1)]$, compared to six LCLs from healthy individuals matched for sex, ethnic origin and age. We used the Affymetrix GeneChip Human Genome U133A 2.0 Arrays, which allowed to screen for 18,400 transcripts including 14,500 wellcharacterized genes.

With Rank Product analysis we obtained a list of 76 statistically significant differentially expressed probes in patients vs. controls, 41 of which were up-regulated (Fold Change - FC $=2.5-10$ ) and 35 down-regulated $(\mathrm{FC}=0.1-0.3)$. These probes corresponded to 66 differentially expressed genes (35 up-regulated and 31 downregulated: one gene may be tested with more than one probe) (Figure 1A) (GEO accession number GSE42406 and Additional file 3). Microarray analysis did not show any statistically significant difference in the mean expression of $A F G 3 L 2$. The ratio between the expression of AFG3L2 and his paralog SPG7 was 1.2 (9.3 vs. 7.7) both in patients and controls, in line with data previously obtained in mice nervous tissues, where $A F G 3 L 2$ is always more expressed than SPG7 [37].

Hierarchical clustering showed that patients' whole genome-expression profiles gathered together, with the exception of patient P3M (mutation p.Met666Val) whose profile was slightly different (Figure 1A).

We used eleven genes to validate microarray data by real-time RT-PCR ( $A D C Y 1$, GPX7, FOXO3, TES, CCDC92, $B L K, F Y N, A T F 5, N O T C H 2, C Y B 5 R 2$, and DPYD) in a total of eight SCA28 patients lines. Expression levels were comparable to those obtained by array profiling (Figure 1B).

The Significance Analysis of Microarrays (SAM), a more stringent statistical method, highlighted five out of the 66 genes resulting from the Rank Product test, all of which up-regulated: Adenylate cyclase 1 (brain) $(A D C Y 1$, $\mathrm{FC}=6.15)$; Glutathione peroxidase $7(G P X 7, \mathrm{FC}=2.85)$; Forkhead box $\mathrm{O} 3$ (FOXO3, $\mathrm{FC}=2.63)$; Testis derived transcript (3 LIM domains) (TES, FC $=1.48$ ); Coiled-coil domain containing $92(C C D C 92, \mathrm{FC}=1.46)$.

To cluster the 66 deregulated genes for function/cellular process we integrated the Gene Ontology (GO) classification with information available through NCBI databank [38]. Five major categories were identified (Figure 1C): (1) regulation of cell proliferation; (2) regulation of programmed cell death; (3) response to oxidative stress; (4) cell adhesion, and (5) chemical homeostasis (more details in Additional file 3).

\section{Cell proliferation, cell cycle and cell viability assays}

Given the deregulation of genes involved in cell proliferation/death, we set up a series of experiments aimed at evaluating these pathways. Using the MTT and Neuro$\mathrm{DiO}$ assays, SCA28 LCLs displayed a reduced growth compared to controls $(\mathrm{p}<0.001)$ (Figure 2A). This observation is supported by the results of the cell cycle analysis that showed an increased percentage of cells 


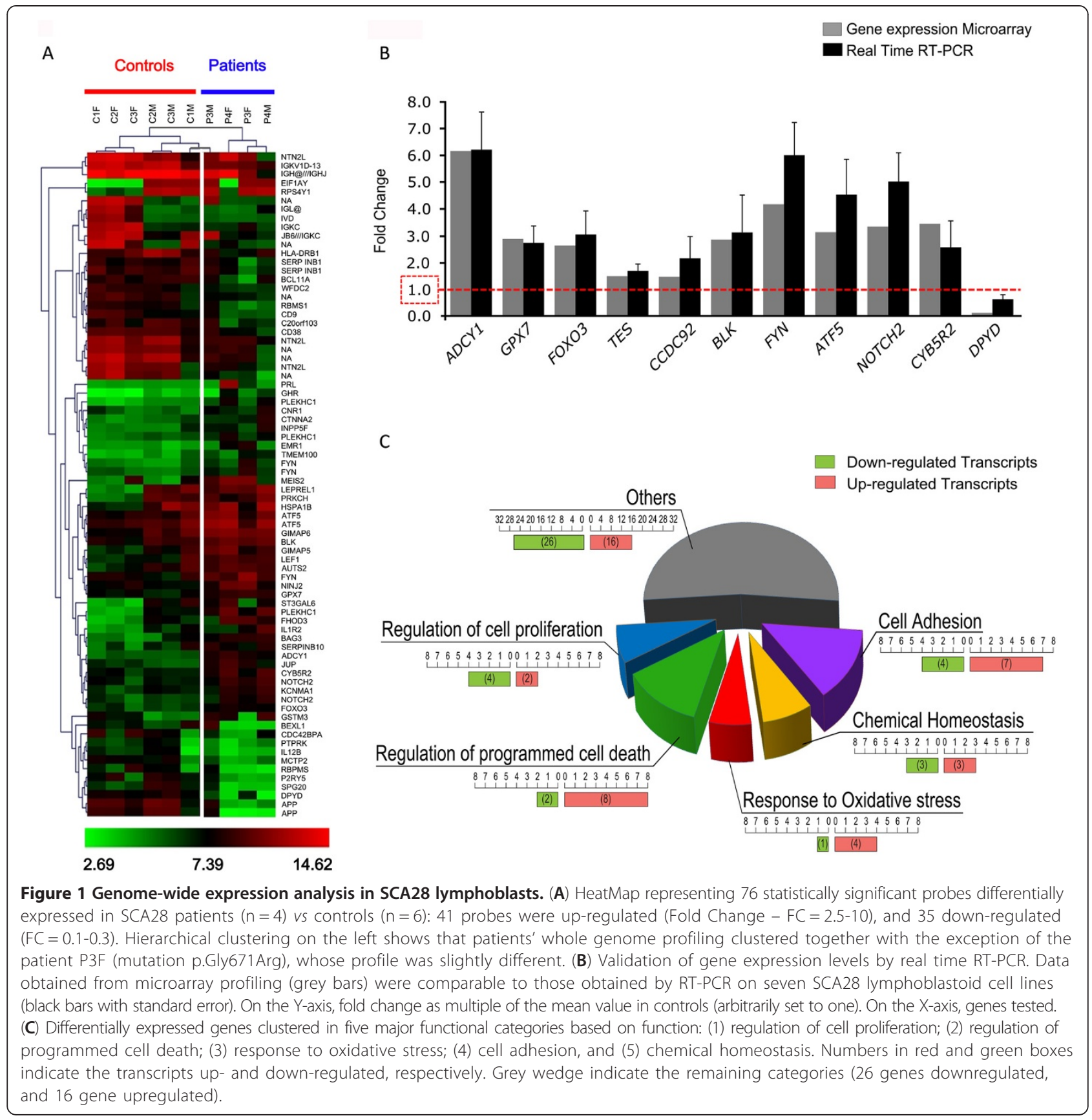

in G0/G1 phase (> 15\%) in SCA28 LCLs compared to controls. The percentage of cells in G0/G1 phase was already different at time $0(72 \pm 1.8 \%$ of SCA28 vs. $59 \pm$ $2.3 \%$ of CTRLs, $\mathrm{p}=0.0003)$ and increased at 3 hours $(68 \pm$ $2.1 \%$ vs. $49 \pm 4.2 \%, \mathrm{p}=0.0006)$, and at 24 hours $(69 \pm 2.1 \%$ vs. $56 \pm 1.4 \%, \mathrm{p}=0.0001)$ after restoration of nutrients (Figure 2B).

Cell viability measured by AnnexinV/Propidium Iodide (PI) double staining showed increased cell death in SCA28 patients with $41 \pm 4.2 \%$ of viable cells $v s .61 \pm 3.6 \%$ in controls LCLs (One-tailed Student's $t$-test, $\mathrm{p}=0.003$; Figure 2C).

\section{Oxidative stress}

Since oxidative stress response was one of the pathways highlighted by the gene expression analysis, we measured direct and indirect signs of ROS increase. Quantitative analysis of intracellular ROS levels with the DCFH-DA test showed that the basal levels of intracellular ROS in SCA28 patients LCLs were comparable to 


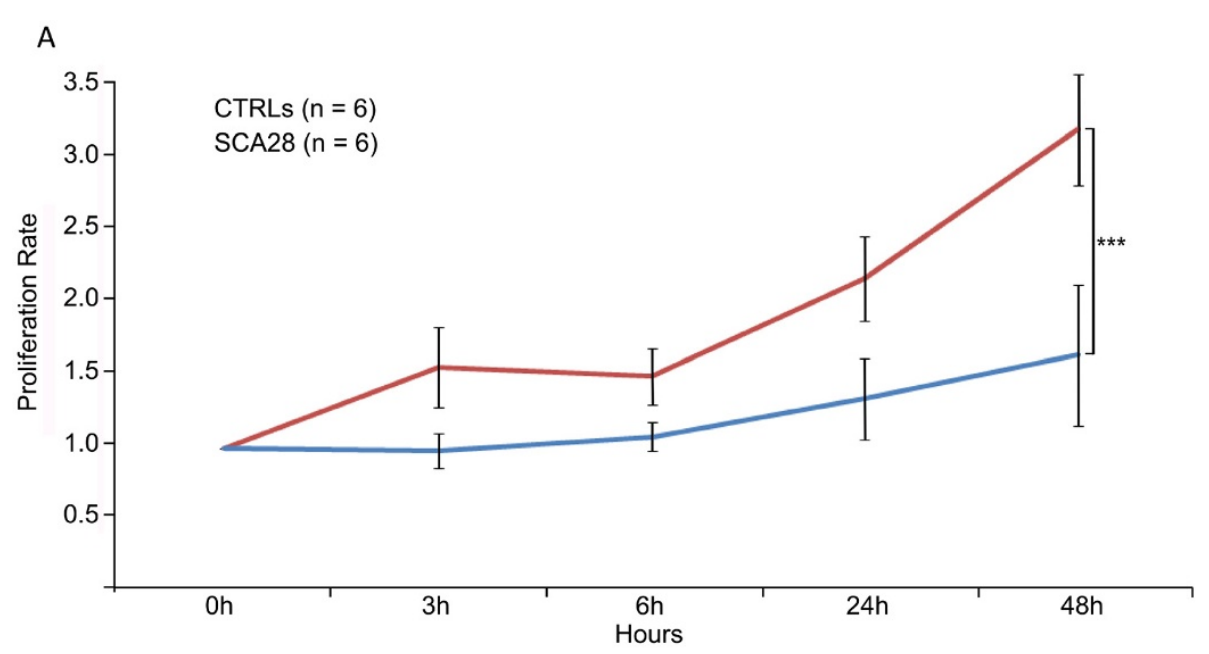

B

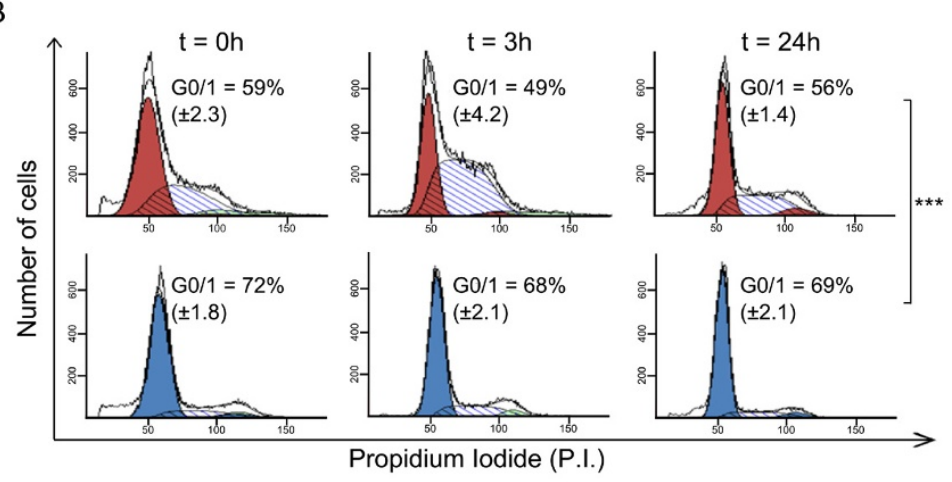

C
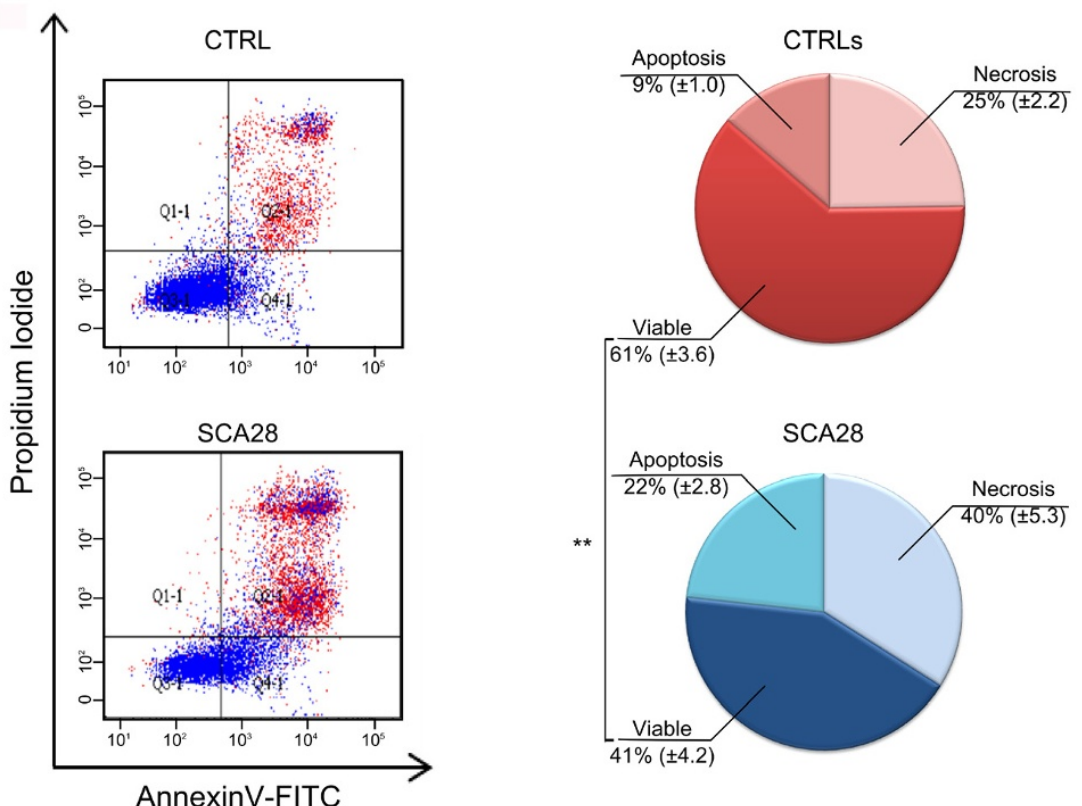

Figure 2 Cell growth, cell cycle and apoptosis in SCA28 LCLs. (A) Using the MTT assay, SCA28 LCLs displayed a reduced growth compared to controls $(p<0.001)$. (B) FACS analysis revealed that patients' LCLs showed an increased number of cells in G0/G1 phase (> 15\%) compared to control LCLs at any time point considered. (C) AnnexinV/PI FACS analysis showed that $41 \pm 4.2 \%$ of SCA28 cells were viable, vs. $61 \pm 3.6 \%$ of control cells (One-tailed Student's t-test, $\mathrm{p}=0.003)$. 
those of healthy controls (data not shown). We evaluated MDA levels as an indirect parameter of intracellular oxidative stress measuring the production of lipid peroxidation markers (TBARS): the results showed a $\sim 2$ fold increased level of TBARS in SCA28 LCLs vs. controls (Kruskal-Wallis test, $\mathrm{p}=0.0014$ ) (Figure 3A). The TBARS levels in SCA28 LCLs were comparable to those reached by control cells after incubation with hydrogen peroxide, a potent inducer of ROS production.

\section{Mitochondrial functionality}

To investigate the effects of AFG3L2 missense changes in mitochondrial metabolism, we analyzed ATP synthesis both in SCA28 cells and in isolated mitochondria in the presence of selected substrates and inhibitors. We did detect neither a significant impairment in ATP synthesis in isolated mitochondria nor a reduction in the total ATP cellular content (data not shown).

Next, we assessed the mtDNA copy number by realtime PCR to exclude mtDNA depletion. No statistically significant differences were detectable in patients compared to controls and no mtDNA deletions or large rearrangements were found (data not shown).

We measured the expression levels of six mitochondrial proteins representative of respiratory chain complexes or regulators of mtDNA replication/transcription (i.e., ATPase- $\alpha$, Core2, NDUFA9, POLG1, SDHA, TFAM, VDAC). Western blot analysis of Mitochondrial Transcription Factor A (TFAM) showed a significant increase of protein levels compared to controls (Figure 3B), whereas no differences were appreciable in the other tested proteins (Additional file 4).

\section{Mitochondrial fission/fusion}

We last explored the fission/fusion network in SCA28 LCLs, measuring DRP1, MFN1, MFN2, and OPA1 protein expression (Additional file 4). Among these, only DRP1 showed a statistically significant reduction in SCA28 LCLs compared to controls (two-tailed Student's $t$-test, $\mathrm{p}=0.02$, Figure $3 \mathrm{C}$ ).

\section{Discussion}

A critical aspect of neurodegenerative disorders studies in humans is that they are hampered by the difficulty in obtaining the pathological tissues. In the attempt to overcome this limitation, other cell types such as fibroblasts, lymphocytes, or immortalized LCLs have been exploited for a wide set of experiments [39,40]. To identify disease pathways and biomarkers for fast diagnoses, microarray genome-wide expression technology, often followed by functional studies, has been used [41].

In this paper, we studied the genomic expression profile of SCA28 patients LCLs and validated the data by real-time PCR, including in a replication set of patients and controls LCLs. Differentially expressed transcripts clustered in five major functional categories: (1) regulation of cell proliferation; (2) regulation of programmed cell death; (3) response to oxidative stress; (4) cell adhesion, and (5) chemical homeostasis. Alterations of these pathways are often reported in neurodegeneration. Functional experiments confirmed an overall cellular metabolic impairment. When compared to healthy subjects, SCA28 LCLs showed an impaired growth, and an increased number of cells in the G0/G1 phase that indicated the activation of the G1 cell cycle checkpoint. Cell cycle alterations have been demonstrated in several CNS diseases including both acute damage and chronic neurodegenerative disorders [42]. Furthermore, it has been shown that the transition between G0/G1 and $S$ phases of the cell cycle is marked by the switch from hyperfused to fragmented mitochondria [43], and mitochondrial fission is dependent on the increase of
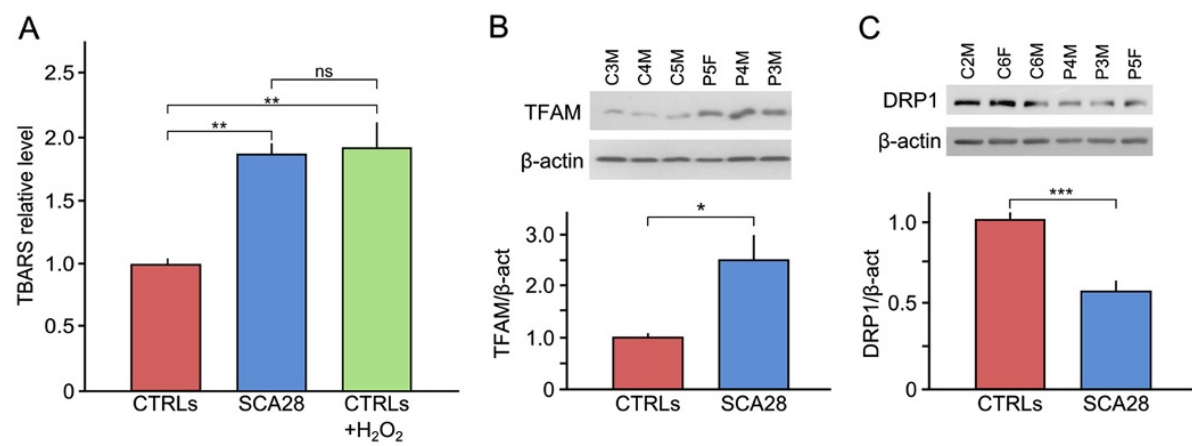

Figure 3 Analysis of lipid peroxidation and mitochondrial function. (A) Thiobarbituric Acid Reactive Substances (TBARS), an indirect marker of ROS was measured in SCA28 LCLs compared to controls (Kruskal-Wallis test, ${ }^{* *}: p=0.0014$; ns: not significative). SCA28 showed a two-fold increase level of TBARS suggesting the presence of hidden ROS damages, that however are not associated with an anomalous respiratory chain activity and total ATP content (not shown). (B) Western blot analysis of TFAM (Mitochondrial Transcription Factor A) protein showed an increase in SCA28 cell lines, suggesting an impairment in mtDNA turnover. (C) The analysis of selected proteins involved in mitochondrial fission/fusion showed a statistical significant reduction in DRP1 in SCA28 LCLs compared to controls LCLs (two-tailed $t$ test $p=0.02$ ). 
DRP1 [44]. It is therefore possible that the decreased DRP1 expression highlighted in SCA28 LCLs may affect the activity of the mitochondrial fusion/fission machinery and may be related to the cell cycle block in the G0/G1 phase.

SCA28 cells showed an increased expression of BAG3, FOXO3 and FYN genes that indicates the attempt to activate an anti-apoptotic response. This, however, was not sufficient to rescue these cells, which are much more prone to undergo apoptotic death that the control cells.

On the other hand, the impairment of the oxidative stress metabolic impairment may underpin the cell cycle anomalies. Accordingly, we found increased expression of $A D C Y 1, F O X O 3$, and GPX7, involved in ROS detoxification [45-47]. Increased and/or impaired ROS response is mainly the outcome of an altered mitochondrial functionality, and fits with the mitochondrial localization of AFG3L2. Moreover, it has been recently demonstrated that defective mitochondrial metabolism is detrimental for Purkinje cells, affected in spinocerebellar ataxia [39]. Intracellular ROS measured using the DCFH-DA were not increased in SCA28 cells, at least in the experimental condition adopted. However, looking at a long lasting damage induced by ROS by means of the TBARS analysis we found increased levels of lipid peroxidation. It is conceivable that the rapid metabolism of LCLs, along with the short half-life of ROS, led to a rapid turnover of oxidative species, masking the direct increase of intracellular ROS but leaving long-term by-products in the form of peroxidated lipids.

Lipid peroxidation of cell membrane phospholipids also leads to the production of several highly reactive carbonyl compounds as by-products, which may form a variety of toxic adducts including cross-linked products on amino acids or DNA bases [48]. Literature data have shown that these products are able to damage mtDNA and key mitochondrial constituents, including enzymes involved in mitochondrial respiration [49]. Nonetheless, we did not detect an altered ATP synthesis in isolated mitochondria or total cell lysates from SCA28 cells, in contrast with loss-of-function $A f g 3 l 2^{E m v 66 / E m v 66}$, $A f g 3 l 2^{E m v 66 /+}$, and $A f g 3 l 2^{\text {par/par }}$ mouse models, where complex I and III deficits were reported [16,17]. Neither altered mtDNA copy number nor mtDNA deletions/rearrangements were furthermore detected in SCA28 LCLs, suggesting either that the level of reactive oxygen species does not trigger major mtDNA damages, or that LCLs turnover in culture does not allow for such genetic damage accumulation. In fact, it must be underlined that LCLs are not the ideal model to investigate mitochondrial functions, due to the small cytoplasmic percentage within cells. Nonetheless, these caveats reinforce the importance of our differential data between cases and controls. Interestingly, a comparable number of mtDNA molecules associated with an increase of TFAM, a crucial protein for mtDNA maintenance, may suggest an impairment in mtDNA turnover leading to the accumulation of its 'histone-like' protein [50,51].

Besides achieving the goal of characterizing SCA28 LCLs cellular hallmarks, our data allow to speculate about a possible pathogenic mechanism of SCA28. It is plausible to think that $A F G 3 L 2$ mutations likely impair the maturation of $m$-AAA substrates and/or the turnover of misfolded proteins leading to a mitochondrial damage (e.g., excess of fission and/or abnormal mtDNA turnover) and to an increased oxidative stress (increased lipid peroxidation). Extracellular stresses, such as starvation or thermal shock and ageing, may worsen this damage. The last chance for the cell is to remove damaged mitochondria "homogenizing" organelle contents through fission/fusion processes, but if this process is impaired (as it is likely in SCA28 LCLs) it drives cells towards death (Figure 4).

Other cell types may respond to an AFG3L2 altered function with a different efficiency and looking at mitochondria as an integrated subcellular system, each aspect of its function can be potentially impaired: bioenergetics, trafficking, organelle interconnectivity (fission/fusion) and protein quality control.

\section{Conclusions}

In conclusion, whole genome expression profiling, performed on SCA28 LCLs, allowed us to identify five altered functional categories that characterize the SCA28 LCLs phenotype, the first reported in human cells to our knowledge.

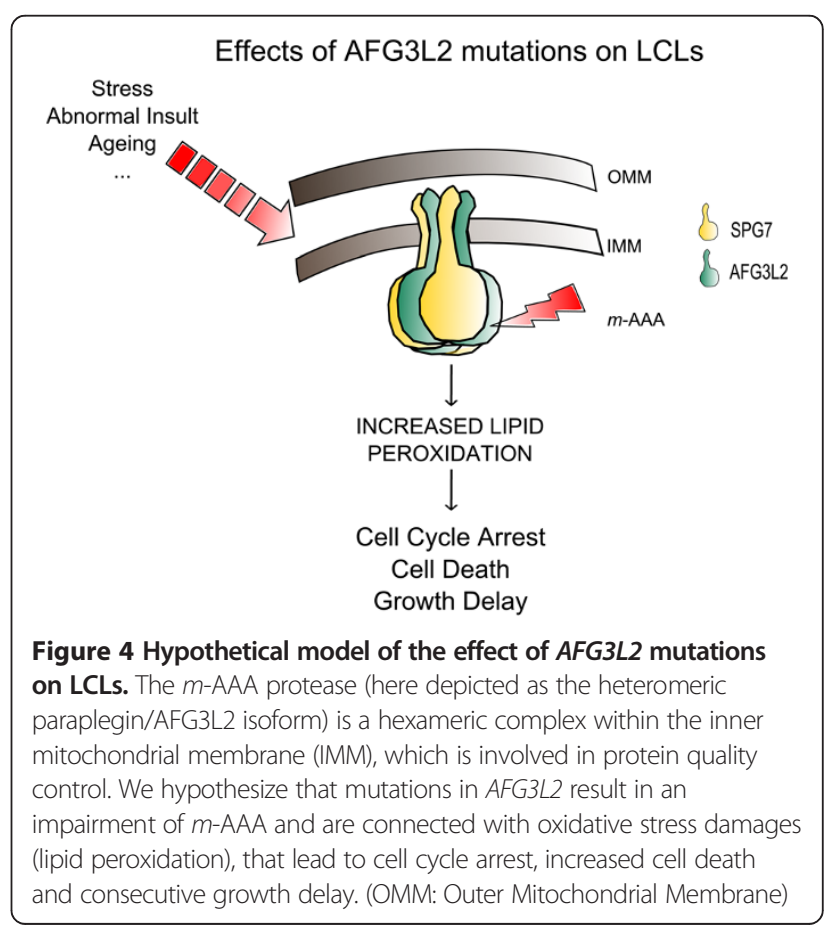


The identification of specific and measurable functional aspects, such as cell cycle blockage, decreased cell growth or increased apoptosis characterizing SCA28 cells, may be used as readout for high-throughput screenings of potential drugs.

\section{Additional files}

\section{Additional file 1: Phenotypic features of SCA28 patients used to obtain lymphoblastoid cell lines (LCLs).}

Additional file 2: Primers and UPL probes used for real-time RT-PCR validation of microarray data.

Additional file 3: Rank product list results and clusters for Gene Ontology categories.

Additional file 4: Western blot analysis of mitochondrial proteins. ATPase-a, Core2, NDUFA9, POLG1, SDHA, VDAC (A) and MFN1, MFN2, and OPA1(B) showed no differences in patients vs. controls.

\section{Competing interests}

The authors declare that they have no competing interests.

\section{Authors' contributions}

CM participated in the conception of the study and in experiments design, carried out the interpretation of expression data, performed the real-time and pathway-validation experiments, and participated in the draft of the manuscript. PR performed genome expression experiments and data analysis, and revised the manuscript. ABrussino participated in the conception of the study and in the draft of the manuscript. GS collected patients' sample and revised the manuscript. NLB analyzed FACS data and revised the manuscript. HK performed array hybridization and revised the manuscript. FM performed ATP synthesis experiments and revised the manuscript. EG performed TBARS experiments and revised the manuscript. ABS performed mitochondrial proteins Western blotting and revised the manuscript. MAC performed total ATP content assay and revised the manuscript. LI evaluated mitochondrial DNA copy number and revised the manuscript. CC participated in in experiments design and in the draft of the manuscript. SF, IL, and AD collected patients' sample and revised the manuscript. AD and ABrice collected patients' clinical data and revised the manuscript. DG participated in TBARS experiment design and data analysis and revised the manuscript. GC participated in ATP synthesis experiment design and data analysis and revised the manuscript. AMP participated in total ATP content/mtDNA copy number experiments design and data analysis and revised the manuscript. AF contributed to cell cycle and cell growth experiments design and data analysis and revised the manuscript. GG contributed to mitochondrial function experiments design and data analysis, and revised the manuscript. SG contributed to genome expression experiments design and data analysis, and revised the manuscript. ABrusco participated in the conception and supervision of the study and in the draft and revision of the manuscript. All authors read and approved the final manuscript.

\section{Acknowledgments}

We are grateful to the patients' family members for their participation, to the DNA and Cell bank of the Centre de Recherche de l'Institut du Cerveau et de la Moelle épinière for technical assistance and to Drs C Marelli, J Yaouang, M Ponsot, H Chneiweiss, D Ménard, S Schaeffer, A Lagueny, L Carluer and Y Boukhriche as well as Prof A Harding for referring some of the patients and for clinical examinations.

This work was funded by Telethon Research grant GGP07110 and GGP12217 (to A Brusco), Regione Piemonte Ricerca Sanitaria Finalizzata, the European Union (to the EUROSCA consortium), the VERUM foundation (to A Brice) and the Programme Hospitalier de Recherche Clinique (to A Durr). We thank prof. G. Matullo (Human Genetics Foundation of Torino) for providing control cell lines.

\section{Author details}

'Department of Medical Sciences, University of Torino, via Santena 19, 10126 Torino, Italy. ${ }^{2}$ European Bioinformatics Institute, Cambridge, UK.
${ }^{3}$ Neurobiology Sector, SISSA/ISAS, Trieste, Italy. ${ }^{4}$ Centre de Recherche de I'Institut du Cerveau et de la Moelle épinière (INSERM / UPMC Univ. Paris 6, UMR_S975 ; CNRS 7225), Pitié-Salpêtrière Hospital, Paris, France. ${ }^{5}$ APHP, Fédération de génétique, Pitié-Salpêtrière Hospital, Paris, France.

${ }^{6}$ Neurogenetics team, Ecole Pratique des Hautes Etudes, Institut du Cerveau et de la Moelle épinière, CHU Pitié-Salpêtrière, Paris, France. ${ }^{7}$ San Raffaele Scientific Institute, Vita-Salute San Raffaele University and Center for Translational Genomics and Bioinformatics, Milan-I, Italy. ${ }^{8}$ Department of Oncology, University of Torino, Candiolo, Italy. ${ }^{9}$ Department Medical and Surgical Sciences, Medical Genetics, University of Bologna, Bologna, Italy. ${ }^{10}$ Department of Pharmacy and Biotechnologies (FABIT), University of Bologna, Bologna, Italy. ${ }^{11}$ Medical Genetics Unit, "Città della Salute e della Scienza" Hospital, Torino, Italy.

Received: 11 February 2013 Accepted: 5 June 2013 Published: 18 June 2013

\section{References}

1. Durr A: Autosomal dominant cerebellar ataxias: polyglutamine expansions and beyond. Lancet Neurol 2010, 9(9):885-894.

2. Cagnoli C, Mariotti C, Taroni F, Seri M, Brussino A, Michielotto C, Grisoli M, Di Bella D, Migone N, Gellera C, et al: SCA28, a novel form of autosomal dominant cerebellar ataxia on chromosome 18p11.22-q11.2. Brain 2006, 129(Pt 1):235-242.

3. Di Bella D, Lazzaro F, Brusco A, Plumari M, Battaglia G, Pastore A, Finardi A, Cagnoli C, Tempia F, Frontali M, et al: Mutations in the mitochondrial protease gene AFG3L2 cause dominant hereditary ataxia SCA28. Nat Genet 2010, 42(4):313-321.

4. Cagnoli C, Stevanin G, Brussino A, Barberis M, Mancini C, Margolis RL, Holmes SE, Nobili M, Forlani S, Padovan S, et al: Missense mutations in the AFG3L2 proteolytic domain account for approximately $1.5 \%$ of European autosomal dominant cerebellar ataxias. Hum Mutat 2010, 31(10):1117-1124.

5. Edener U, Wollner J, Hehr U, Kohl Z, Schilling S, Kreuz F, Bauer P, Bernard V, Gillessen-Kaesbach $G$, Zuhlke $C$ : Early onset and slow progression of SCA28, a rare dominant ataxia in a large four-generation family with a novel AFG3L2 mutation. Eur J Hum Genet 2010, 18(8):965-968.

6. Banfi S, Bassi MT, Andolfi G, Marchitiello A, Zanotta S, Ballabio A, Casari G, Franco B: Identification and characterization of AFG3L2, a novel paraplegin-related gene. Genomics 1999, 59(1):51-58.

7. Leonhard K, Stiegler A, Neupert W, Langer T: Chaperone-like activity of the AAA domain of the yeast Yme1 AAA protease. Nature 1999, 398(6725):348-351.

8. Arlt $H$, Tauer R, Feldmann $H$, Neupert W, Langer T: The YTA10-12 complex, an AAA protease with chaperone-like activity in the inner membrane of mitochondria. Cell 1996, 85(6):875-885.

9. Leonhard K, Guiard B, Pellecchia G, Tzagoloff A, Neupert W, Langer T: Membrane protein degradation by AAA proteases in mitochondria: extraction of substrates from either membrane surface. Mol Cell 2000, 5(4):629-638.

10. Nolden M, Ehses S, Koppen M, Bernacchia A, Rugarli El, Langer T: The m-AAA protease defective in hereditary spastic paraplegia controls ribosome assembly in mitochondria. Cell 2005, 123(2):277-289.

11. Koppen M, Bonn F, Ehses S, Langer T: Autocatalytic processing of m-AAA protease subunits in mitochondria. Mol Biol Cell 2009, 20(19):4216-4224.

12. Gerdes F, Tatsuta T, Langer T: Mitochondrial AAA proteases - Towards a molecular understanding of membrane-bound proteolytic machines. Biochim Biophys Acta 2011, 1823(1):49-55.

13. Casari G, De Fusco M, Ciarmatori S, Zeviani M, Mora M, Fernandez P, De Michele G, Filla A, Cocozza S, Marconi R, et al: Spastic paraplegia and OXPHOS impairment caused by mutations in paraplegin, a nuclearencoded mitochondrial metalloprotease. Cell 1998, 93(6):973-983.

14. Pierson TM, Adams D, Bonn F, Martinelli P, Cherukuri PF, Teer JK, Hansen NF, Cruz P, Mullikin For The Nisc Comparative Sequencing Program JC, Blakesley RW, et al: Whole-exome sequencing identifies homozygous AFG3L2 mutations in a spastic ataxia-neuropathy syndrome linked to mitochondrial m-AAA proteases. PLoS Genet 2011, 7(10):e1002325.

15. Klebe S, Depienne C, Gerber S, Challe G, Anheim M, Charles P, Fedirko E, Lejeune E, Cottineau J, Brusco A, et al: Spastic paraplegia gene 7 in patients with spasticity and/or optic neuropathy. Brain 2012, 135(Pt 10):2980-2993. 
16. Maltecca F, Aghaie A, Schroeder DG, Cassina L, Taylor BA, Phillips SJ, Malaguti M, Previtali S, Guenet JL, Quattrini A, et al: The mitochondrial protease AFG3L2 is essential for axonal development. J Neurosci 2008, 28(11):2827-2836

17. Maltecca F, Magnoni R, Cerri F, Cox GA, Quattrini A, Casari G: Haploinsufficiency of AFG3L2, the gene responsible for spinocerebellar ataxia type 28 , causes mitochondria-mediated Purkinje cell dark degeneration. J Neurosci 2009, 29(29):9244-9254.

18. Bioconductor. http://www.bioconductor.org.

19. MultiExperiment Viewer. http://www.tm4.org/mev/.

20. Breitling R, Armengaud P, Amtmann A, Herzyk P: Rank products: a simple, yet powerful, new method to detect differentially regulated genes in replicated microarray experiments. FEBS Lett 2004, 573(1-3):83-92.

21. Tusher VG, Tibshirani R, Chu G: Significance analysis of microarrays applied to the ionizing radiation response. Proc Natl Acad Sci USA 2001, 98(9):5116-5121.

22. Ashburner M, Ball CA, Blake JA, Botstein D, Butler H, Cherry JM, Davis AP, Dolinski K, Dwight SS, Eppig JT, et al: Gene ontology: tool for the unification of biology, The Gene Ontology Consortium. Nat Genet 2000, 25(1):25-29

23. da Huang W, Sherman BT, Lempicki RA: Systematic and integrative analysis of large gene lists using DAVID bioinformatics resources. Nat Protoc 2009, 4(1):44-57.

24. da Huang W, Sherman BT, Lempicki RA: Bioinformatics enrichment tools: paths toward the comprehensive functional analysis of large gene lists. Nucleic Acids Res 2009, 37(1):1-13.

25. Dennis G Jr, Sherman BT, Hosack DA, Yang J, Gao W, Lane HC, Lempicki RA: DAVID: Database for Annotation, Visualization, and Integrated Discovery. Genome Biol 2003, 4(5):P3.

26. Livak KJ, Schmittgen TD: Analysis of relative gene expression data using real-time quantitative PCR and the 2(-Delta Delta $C(T))$ Method. Methods 2001, 25(4):402-408.

27. Mosmann T: Rapid colorimetric assay for cellular growth and survival: application to proliferation and cytotoxicity assays. J Immunol Methods 1983, 65(1-2):55-63.

28. Cunningham RE: Overview of flow cytometry and fluorescent probes for flow cytometry. Methods Mol Biol 2010, 588:319-326.

29. Vermes I, Haanen C, Steffens-Nakken H, Reutelingsperger C: A novel assay for apoptosis, Flow cytometric detection of phosphatidylserine expression on early apoptotic cells using fluorescein labelled Annexin V. $\mathrm{J}$ Immunol Methods 1995, 184(1):39-51.

30. Brumatti G, Sheridan C, Martin SJ: Expression and purification of recombinant annexin $\mathrm{V}$ for the detection of membrane alterations on apoptotic cells. Methods 2008, 44(3):235-240.

31. Robinson $\mathrm{BH}$ : Use of fibroblast and lymphoblast cultures for detection of respiratory chain defects. Methods Enzymol 1996, 264:454-464.

32. Ronner P, Friel E, Czerniawski K, Frankle S: Luminometric assays of ATP, phosphocreatine, and creatine for estimation of free ADP and free AMP. Anal Biochem 1999, 275(2):208-216.

33. Zanna C, Ghelli A, Porcelli AM, Martinuzzi A, Carelli V, Rugolo M: Caspaseindependent death of Leber's hereditary optic neuropathy cybrids is driven by energetic failure and mediated by AIF and Endonuclease G. Apoptosis 2005, 10(5):997-1007.

34. Yano E: Mineral fiber-induced malondialdehyde formation and effects of oxidant scavengers in phagocytic cells. Int Arch Occup Environ Health 1988, 61(1-2):19-23.

35. Iommarini L, Maresca A, Caporali L, Valentino ML, Liquori R, Giordano C, Carelli V: Revisiting the issue of mitochondrial DNA content in optic mitochondriopathies. Neurology 2012, 79(14):1517-1519.

36. Kleinle S, Wiesmann U, Superti-Furga A, Krahenbuhl S, Boltshauser E, Reichen J, Liechti-Gallati S: Detection and characterization of mitochondrial DNA rearrangements in Pearson and Kearns-Sayre syndromes by long PCR. Hum Genet 1997, 100(5-6):643-650

37. Sacco T, Boda E, Hoxha E, Pizzo R, Cagnoli C, Brusco A, Tempia F: Mouse brain expression patterns of Spg7, Afg3l1, and Afg312 transcripts, encoding for the mitochondrial m-AAA protease. BMC Neurosci 2010, 11:55.

38. National Center for Biotechnology Information. http://www.ncbi.nlm.nih.gov

39. Yan H, Yuan W, Velculescu VE, Vogelstein B, Kinzler KW: Allelic variation in human gene expression. Science 2002, 297(5584):1143.
40. Cheung VG, Conlin LK, Weber TM, Arcaro M, Jen KY, Morley M, Spielman RS: Natural variation in human gene expression assessed in lymphoblastoid cells. Nat Genet 2003, 33(3):422-425.

41. Cooper-Knock J, Kirby J, Ferraiuolo L, Heath PR, Rattray M, Shaw PJ: Gene expression profiling in human neurodegenerative disease. Nat Rev Neurol 2012, 8(9):518-530

42. Yang Y, Herrup K: Cell division in the CNS: protective response or lethal event in post-mitotic neurons? Biochim Biophys Acta 2007, 1772(4):457-466

43. Margineantu DH, Gregory Cox W, Sundell L, Sherwood SW, Beechem JM, Capaldi RA: Cell cycle dependent morphology changes and associated mitochondrial DNA redistribution in mitochondria of human cell lines. Mitochondrion 2002, 1(5):425-435

44. Parone PA, Da Cruz S, Tondera D, Mattenberger $Y$, James DI, Maechler $P$, Barja F, Martinou JC: Preventing mitochondrial fission impairs mitochondrial function and leads to loss of mitochondrial DNA. PLOS One 2008, 3(9):e3257.

45. Utomo A, Jiang X, Furuta S, Yun J, Levin DS, Wang YC, Desai KV, Green JE, Chen $\mathrm{PL}$, Lee $\mathrm{WH}$ : Identification of a novel putative non-selenocysteine containing phospholipid hydroperoxide glutathione peroxidase (NPGPx) essential for alleviating oxidative stress generated from polyunsaturated fatty acids in breast cancer cells. J Biol Chem 2004, 279(42):43522-43529.

46. Masmoudi-Kouki O, Douiri S, Hamdi Y, Kaddour H, Bahdoudi S, Vaudry D, Basille M, Leprince J, Fournier A, Vaudry $H$, et al: Pituitary adenylate cyclase-activating polypeptide protects astroglial cells against oxidative stress-induced apoptosis. J Neurochem 2011, 117(3):403-411.

47. Ferber EC, Peck B, Delpuech O, Bell GP, East P, Schulze A: FOXO3a regulates reactive oxygen metabolism by inhibiting mitochondrial gene expression. Cell Death Differ 2012, 19(6):968-979.

48. Hruszkewycz AM: Evidence for mitochondrial DNA damage by lipid peroxidation. Biochem Biophys Res Commun 1988, 153(1):191-197.

49. Sultana R, Perluigi M, Allan Butterfield D: Lipid peroxidation triggers neurodegeneration: A redox proteomics view into the Alzheimer disease brain. Free Radic Biol Med 2012, S0891-5849(12):01161-01166.

50. Kang D, Kim SH, Hamasaki N: Mitochondrial transcription factor A (TFAM): roles in maintenance of mtDNA and cellular functions. Mitochondrion 2007, 7(1-2):39-44.

51. Noack H, Bednarek T, Heidler J, Ladig R, Holtz J, Szibor M: TFAM-dependent and independent dynamics of mtDNA levels in C2C12 myoblasts caused by redox stress. Biochim Biophys Acta 2006, 1760(2):141-150.

doi:10.1186/1755-8794-6-22

Cite this article as: Mancini et al:: Genome-wide expression profiling and functional characterization of SCA28 lymphoblastoid cell lines reveal impairment in cell growth and activation of apoptotic pathways. BMC Medical Genomics 2013 6:22

\section{Submit your next manuscript to BioMed Central and take full advantage of:}

- Convenient online submission

- Thorough peer review

- No space constraints or color figure charges

- Immediate publication on acceptance

- Inclusion in PubMed, CAS, Scopus and Google Scholar

- Research which is freely available for redistribution 